\title{
Piecing together prehistoric life: Scanning and articulating gorgosaurus
}

Manisha Saraswat ${ }^{1}$, Samantha M. Hamilton ${ }^{1}$, Nicole Jaremco ${ }^{1}$, Howard Gibbins ${ }^{1}$, Clive Coy ${ }^{1}$, Phil Currie ${ }^{1}$

${ }^{1}$ Department of Biological Sciences, University of Alberta

\begin{abstract}
The Skull bones of a Gorgosaurus Libratus was laser scanned in order to articulate the model into software and 3D print. The model had to be articulated due to some missing bone, making it unrealistic to put together. Using the scanned pieces we articulated the model making a skull of The Gorgosaurus Libratus. This detailed computer skull can be sent anywhere in the world, for anyone to study. These scans could also be used to find out how the Gorgosaurus Libratus bit down or determine the way these animals moved. Prior to laser scanning, a method known as Photogrammetry was used. This method involves taking photos of the model and processing the images on a computer, which slow down the process. Another way used to replicate bones was by making silicone molds. This could damage the bone which makes it a method used less often. Laser scanning is the fastest and safest method in order to scan a bone. After the bones were articulated on the computer they were sent to a 3D printer. Unfortunately, the printer beds could not hold the massive skull. Due to this, the bones were printed half size. In order to 3D print, the holes of the model had to be filled using another program. The holes were caused by the light of the laser scanner not being able to go into all the holes creating shadows that the laser scanner could not pick up. However, after the holes were filled some of the objects were still too big to fit on the printer bed. Therefore, some of the objects were cut in half to fit. The 3D printed models were then printed and assembled.
\end{abstract}

Key words:

scanning, articulating, prehistoric life, gorgosaurus, skull, dinosaur provincial park, dinosaur skull bones, bones, fossils, piecing together prehistoric life: scanning and articulating gorgosaurus, Manisha Saraswat, manisha saraswat, WISEST, poster, putting together a skull

Cite as: Saraswat M., M. Hamilton SM., Jaremco N., Gibbins H., Coy C., Currie P. 2019. Piecing Together Prehistoric Life: Scanning and Articulating Gorgosaurus. Alberta Academic Review, Vol 2 (2) 65-66, WISEST Special Issue (non peer-reviewed), DOI 10.29173/aar68. 


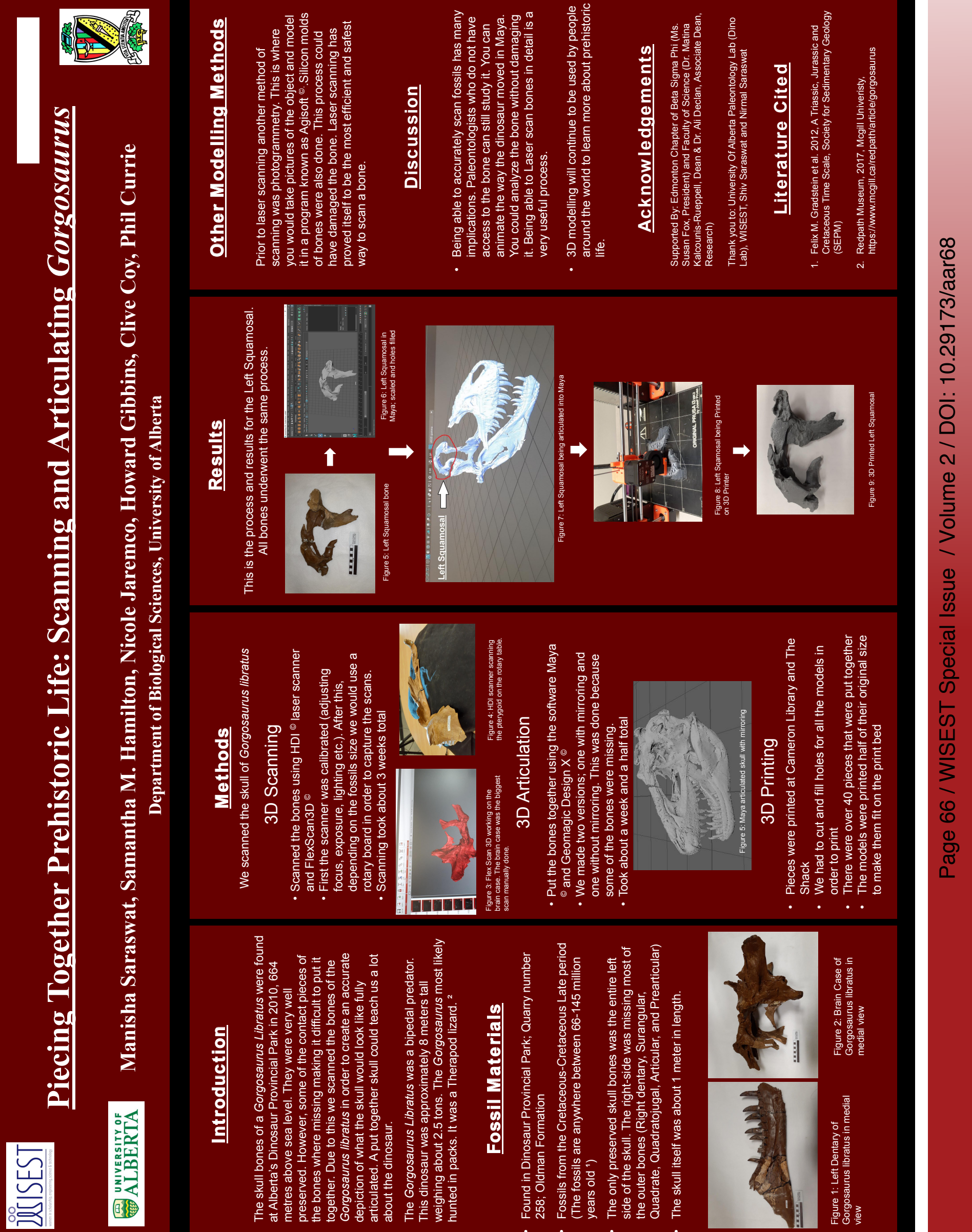

